\title{
Ambient Gesture-Recognizing Surfaces with Visual Feedback
}

\author{
Tobias Grosse-Puppendahl ${ }^{1}$, Sebastian Beck ${ }^{2}$, Daniel Wilbers ${ }^{1}$, Steeven Zeiss ${ }^{1}$, \\ Julian von Wilmsdorff ${ }^{1}$, and Arjan Kuijper ${ }^{1,2}$ \\ 1 Fraunhofer IGD, Fraunhoferstr. 5, 64283 Darmstadt, Germany \\ \{tobias.grosse-puppendahl, daniel.wilbers, steeven.zeiss, \\ julian.von-wilmsdorff, arjan.kuijper\}@igd.fraunhofer.de \\ 2 Technische Universität Darmstadt, Hochschulstr. 10, 64289 Darmstadt, Germany \\ s.beck@stud.tu-darmstadt.de
}

\begin{abstract}
In recent years, gesture-based interaction gained increasing interest in Ambient Intelligence. Especially the success of camera-based gesture recognition systems shows that a great variety of applications can benefit significantly from natural and intuitive interaction paradigms. Besides camera-based systems, proximity-sensing surfaces are especially suitable as an input modality for intelligent environments. They can be installed ubiquitously under any kind of non-conductive surface, such as a table. However, interaction barriers and the types of supported gestures are often not apparent to the user. In order to solve this problem, we investigate an approach which combines a semi-transparent capacitive proximity-sensing surface with an LED array. The LED array is used to indicate possible gestural movements and provide visual feedback on the current interaction status. A user study shows that our approach can enhance the user experience, especially for inexperienced users.
\end{abstract}

Keywords: gesture recognition, capacitive sensing, proximity sensing

\section{Introduction}

Society is very inhomogeneous in itself - possible users' backgrounds differ vastly in age, education, household income and technology experience. Nowadays, technological progress is more rapidly changing than it has ever before and complexity raises simultaneously with growth. The generation of people that did not grow up with computers or touch technology often has difficulties in their first steps of using new technology. Younger people experience difficulties, too, but they are more used to finding their way around these mediums and can generally adapt to new technology faster.

Gesture recognition is a highly promising technology in the field of smart environments, however, it requires training and instruction beforehand. Considering a user's capability to remember gestural movements and the sheer number of interfaces already in use today, high demands arise in terms of cognitive ability. Feedforward and feedback mechanisms for gesture recognition devices may simplify a user's interaction with components and help with memorization. 

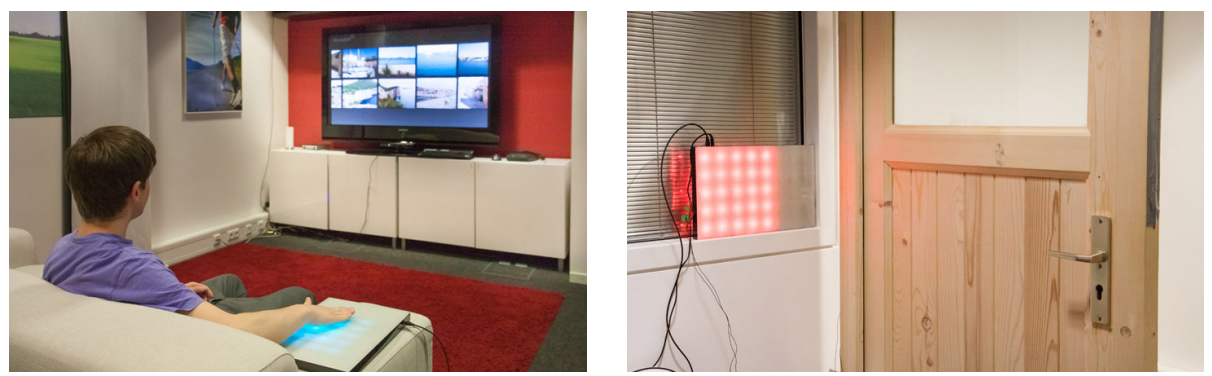

Fig. 1. Low-cost gesture-recognizing surfaces with visual feedback can be employed to control a wide variety of devices within an intelligent environment - reaching from interaction with an entertainment system (left) to interaction with a smart door that can be controlled via gestures, for example in public restrooms (right).

Data acquisition for gesture recognition can rely on different sensing technologies, such as cameras. In our paper, we investigate an alternate low-cost data acquisition approach - an interactive surface equipped with capacitive proximity sensors. It is able to sense hand gestures at distances of up of $20 \mathrm{~cm}$. Rainbowfish' surface can illuminate its surface in different colors, as shown in Figure 1.

Capacitive proximity-sensing gesture recognition systems are able to detect the position of a human body part by combining measurements of many sensors. Each sensor is a combination of a measuring circuit and a sensing electrode made of a variety of materials, depending on the application and the required resolution. An array of capacitive sensors can be used to detect passive interaction patterns, e.g. a person's presence, but also explicit interaction, for example gestures performed by a user over an equipped surface. Besides the low cost compared to cameras, the sensing modality is very suitable in this domain, as sensors can be easily integrated and hidden in walls or furniture $[5,16,11]$. Moreover, we argue that a user's perception of privacy is higher compared to cameras.

Every gesture recognition system faces the challenge of exposing its affordances and providing feedback to a user. For example, users new to a specific system are often not aware of the supported interaction methods. Therefore, it is necessary to deliver interactive feedback on the interaction status and feedforward information about the gestures a user is able to perform. Figure 1 shows our gesture-recognition device projecting a visual feedback directly on its surface. For example, a glowing shadow can follow a user's hand to make interaction barriers more apparent. Moving arrows or stripes can indicate the possibility of performing a horizontal swipe gesture into a certain direction.

In summary, we provide the following contributions:

1. We present a new capacitive gesture recognition system with visual feedback.

2. In a detailed user study we evaluate the approach for its applicability in smart environments.

3. We identify new interaction paradigms and applications that can be realized with the presented technology. 
The remainder of this paper is structured as follows: First, Section 2 deals with related work considering capacitive sensing and gesture recognition in intelligent environments. We present our custom-built hardware in Section 3. The user study and the experimental setup is described in Section 4. We conclude the paper with a summary of our findings in the evaluation and identify potential future research activities.

\section{Related Work}

The success of gesture-based interaction in the context of entertainment systems initiated an increasing trend towards natural interaction paradigms in intelligent environments. Gesture recognition systems in smart environments represent an intuitive and easy way of interaction with the surrounding, for example allowing the user to control everyday devices using simple gestures. There are several technologies that are suitable to act as an input modality for gesture recognition. Commercial camera-based systems, such as the Microsoft Kinect [9], are able to capture gestures and movements within a room. Other approaches employ the environmental noise in an environment [3] or use mobile phones for gesture recognition [1]. Stationary installed capacitive sensors can act as both, touchsensitive and proximity-sensitive gesture-recognizing input modalities. They can be used to detect the way we touch objects [11], or infer hand positions in proximities of up to $40 \mathrm{~cm}[5]$.

Capacitive sensing is a fairly old and well established technology. The sensing principle was firstly applied by a Russian physicist called Leon Theremin in the 1920s [4]. Later, in the 1990s, capacitive proximity sensing was employed to create 3D user interfaces like the LazyFish [12]. These user interfaces are able to recognize objects like human hands within a proximity of up to $50 \mathrm{~cm}[12,5]$.

Today, capacitive sensing found its way into many applications in intelligent environments. Sato et al. use swept frequency capacitive sensing to identify human activities by the way a person touches an object [10]. Especially in smart environments, this approach can recognize how a person touches an object, for example a smart door knob that triggers locking and unlocking of a door [11]. Moreover, the technique can be employed to identify users at the time they touch a screen [7]. Recent activities also include analyzing electro-magnetic noise from household devices to recognize gestures [3].

Proximity sensors based on capacitive sensing are especially suitable for recognizing user interactions within a well-defined interaction space. For example, stationary installed capacitive sensors underneath the floor were used to realize floor localization systems $[15,14]$. Moreover, capacitive sensors deployed in furniture can be used to build interactive applications, for example by recognizing interactions above a table [16]. Recently, an open-source toolkit for rapid prototyping of capacitive sensing applications was presented, that can be used to realize scenarios like 3D gesture recognition, localization and fall detection [5]. Using this toolkit, objects such as human hands can be recognized within a distance of up to $50 \mathrm{~cm}$, depending on the electrode size. 


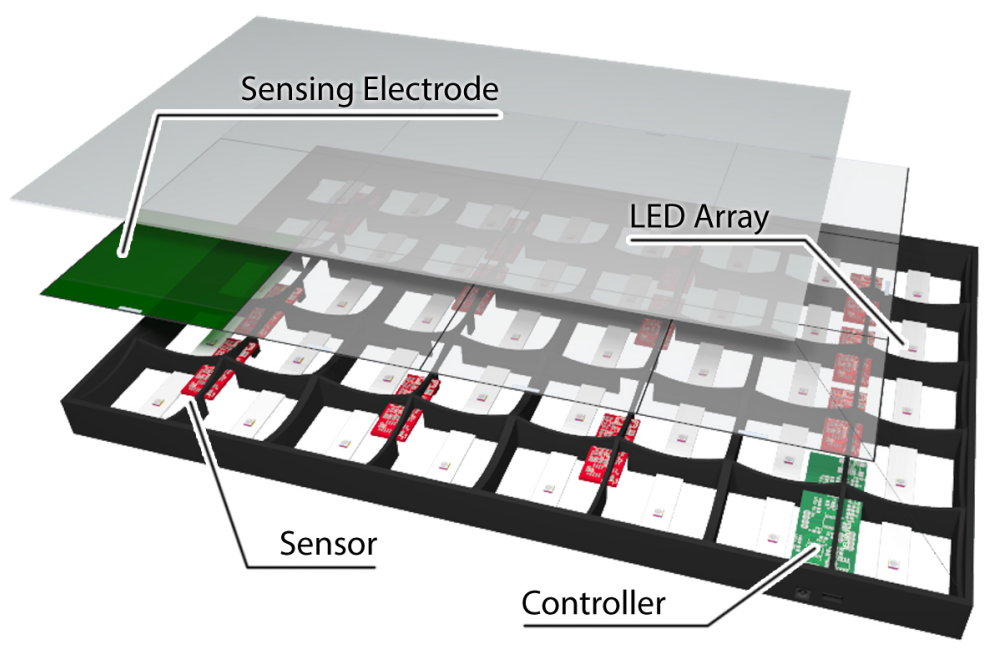

Fig. 2. The device consists of four main components: the sensors, the shielded electrodes made of transparent ITO, an LED array and a controller board. All components are interconnected by an $\mathrm{I} 2 \mathrm{C}$ bus.

In order to extract information from low-level capacitive proximity sensing data an object recognition method is essential. These methods range from fast geometric processing [2] to sophisticated object recognition methods like SwissCheese Extended [6].

Considering scenarios that require gesture recognition without a graphical user interface, it is challenging to provide a suitable and meaningful feedback on the current interaction state to the user. This feedback can be provided by different modalities, for example visually or acoustically. Majewski et al. use a laser spot that visualizes a user's pointing direction perceived by the environment to disambiguate device selection [8]. When a device is selected, the spot delivers additional feedback on the successful selection by blinking. The authors of [13] project a visual feedback directly on the user's body. The presented system provides hints on recommended hand movements and delivers feedback on the movements performed. To our knowledge, capacitive proximity sensing devices have not been directly augmented with visual techniques to give feedback on the current interaction status and indicate possible interaction paradigms.

\section{Hardware \& Processing Chain}

A conceptual drawing of Rainbowfish is depicted in Figure 2. It employs 12 sensors which measure the capacitance between the sensor's electrode and its surroundings, also known as a loading-mode measurement [12]. The sensing electrode's surface builds up an electric field to any object in its surrounding. When a human hand approaches the sensing electrode, the capacitance increases. This 
effect allows for determining an approximate hand distance based on each sensor's measurement. By combining measurements of all 12 sensors, the hand's position can be inferred by triangulation or weighted-average calculations. In order to conduct the measurement, the resulting capacitor between the electrode and the surrounding objects is charged and discharged with a frequency of $500 \mathrm{KHz}$. The sensing electrodes are transparent PET foils with a conductive layer of Indium-Tin-Oxide (ITO), a material widely used in modern capacitive touchscreens. They have a size of $10 \mathrm{~cm}$ by $8 \mathrm{~cm}$ and consist of two layers: a sensing and a shielding layer. The shielding layer is necessary to avoid electronic interferences with the underlying hardware, such as the sensors and the LED array. All components are embedded into a 3D-printed grid structure. The electrodes are adhered underneath the device's top surface - a $3 \mathrm{~mm}$ thick layer of semi-transparent Plexiglas.

The device has a central Inter-Integrated-Circuit (I2C) bus used for interconnecting the sensors with a controller board. This controller board is responsible for scheduling the sensors and controlling the LED array. The measurements are performed concurrently to achieve a suitable temporal and spatial performance. However, when sensor electrodes are located side-by-side, a parallel measurement would affect the neighbouring sensor. Therefore, in each measurement step only three sensors are activated in parallel to avoid interference. Using this method, we currently obtain 20 measurements per second for each sensor. Rainbowfish's controller sends the sensor values to a PC through a USB connection. The PC executes additional processing steps, like drift compensation and normalization, and determines the position of a user's hand. Based on this information, an application is able to send information on its execution state or supported gestures back to Rainbowfish. Instead of sending pixel-related data with a high update rate, the application sends lightweight function calls to trigger pre-defined visualization profiles. These profiles include illuminating the whole surface in a specified color and for a certain time, animating a swiping gesture, drawing colored rectangles, and glow effects on continuous 2D coordinates.

\section{User Study}

The overall goal of the user study is to explore the applicability of visual feedback on a gesture-recognizing surface in smart environments. We stated a number of hypothesis, which were investigated in the study: (H1) visual feedback increases the interaction speed, (H2) a novice user is able to handle an unknown system more easily, (H3) a user is able to recognize usage and system errors immediately, and $\left(H_{4}\right)$ the perception of visual feedback depends on the familiarity with the system. Therefore, we conducted a user study with 18 participants. The study consisted of four main parts: (1) which gestures would a user perform to trigger a certain action, (2) imitate gestures based on visual feedforward animations, (3) interpret visual feedback and (4) use Rainbowfish in two exemplary applications for smart environments. 


\subsection{Perception of Feedback and Feedforward Visualizations}

In order to investigate if users are able to handle a system more easily with visual feedback and feedforward clues (H2), we conducted an experiment consisting of two parts. First, the participants were asked to perform certain gestures to reach an application specific goal without any animations shown on Rainbowfish's service - for example by giving instructions like 'raise the volume of a media player' or 'switch the light off'.

The variety in which the gestures were carried out turned out to be very high and coherent. However, they showed substantial analogies to smartphone and tablet PC usage. Eventually some general statements can be made for certain goals, for example for instructions like select an object. This instruction mainly resulted in grabbing, tapping on the device's surface, or hovering over the object. When considering smart environments, it can be concluded that gesturerecognizing surfaces are very hard to handle without feedforward information if only the functional goals are known to a user.
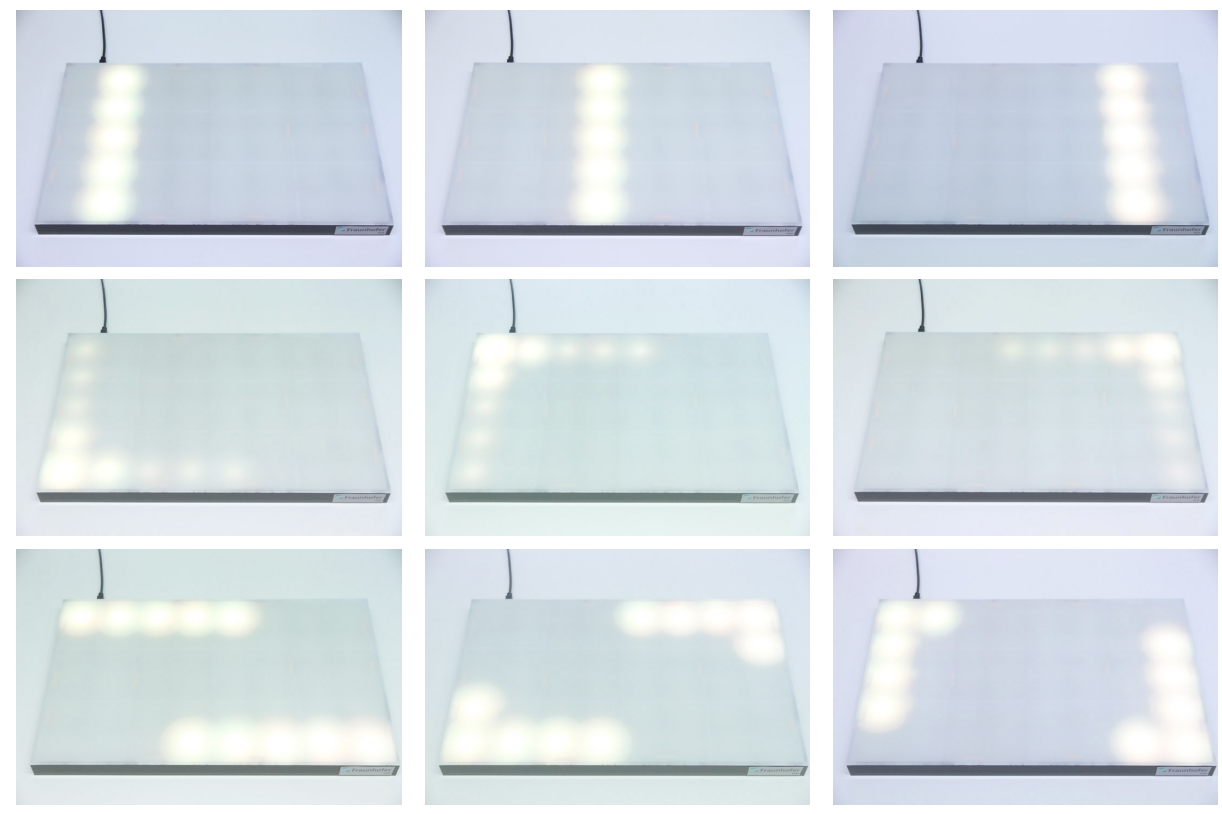

Fig. 3. Feedforward animations for gestures in front of Rainbowfish. The first column shows a swipe gesture from left to right, the second indicates a rotate gesture with a single hand, whereas the third visualization shows a two-handed rotate gesture.

In the second experiment, the test persons were asked to imitate gestures based on feedforward visualizations projected on the Rainbowfish's surface. Again, we investigated the variety of gestures which were carried out. The feedforward animations are shown in Figure 3. Therefore, we exploited analogies to common 
touchscreen gestures (pinch-to-zoom, rotate, etc.), which led to a vast majority of correctly performed gestures (93.5\%). This supports the assumption that the presented feedforward animations are a suitable way of representing the affordances of a gesture-recognizing surface.

In the following experiment, we presented each participant a number of feedback expressions displayed on Rainbowfish's surface. This experiment was conducted to explore how visual feedback provided by an application is perceived by a user (H3). Figure 4 shows a subset of feedback animations which were evaluated. As expected, a short green flash was associated with the acknowledgement of an action by almost all users. On the other hand, a red flash was associated to neglection or rejection. Yellow and blue flashs were mainly associated to a wide variety of meanings, such as waiting or in progress, which does not allow for any generalizable statement. Interestingly, more users were able to associate a green flash with a positive outcome when the complementary red flash was shown afterwards.


Fig. 4. Different types of feedback can be used to indicate certain application-specific outcome. In our study we asked the users to associate a meaning to the animations shown in the three images.

\subsection{Evaluation of Applications in Smart Environments}

In the next part of our user study, we investigated to exemplary applications which we developed for Rainbowfish. In the first application the participants controlled a home entertainment application - an image viewer - with gestures. This application consists of our gesture recognition device, as well as a screen for displaying the images. The second experiment solely employs the gesture recognition device without providing an additional graphical user interface. In this part of the evaluation, the users were asked to open, close and lock an automatic door by performing gestures.

Home Entertainment In this experiment, an image viewer as an exemplary home entertainment application was evaluated. We placed our gesture recognition device in front of a screen that showed the image viewer application. In this setup, depicted in Figure 5, the user is able to manipulate the application's cursor by the position of her or his hands. The participant is able to scroll to 

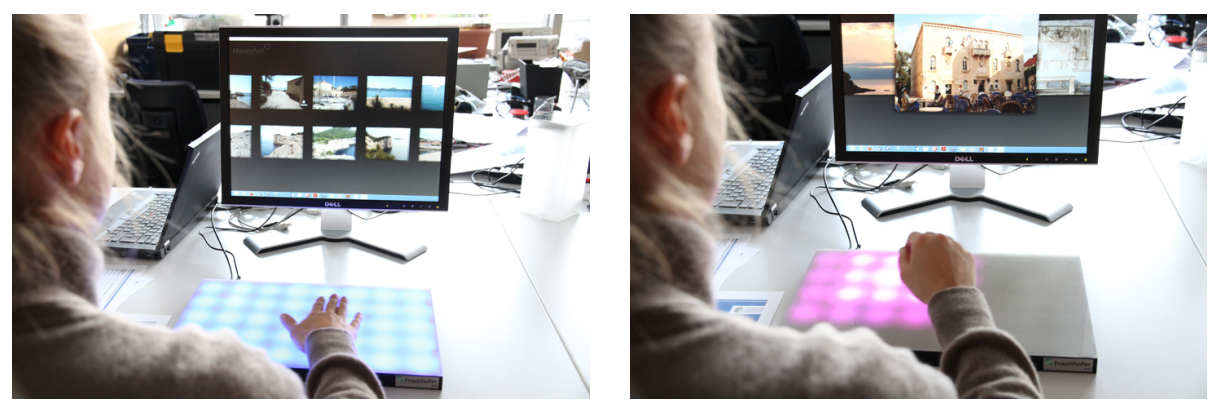

Fig. 5. In the image viewer application, a user is able to select and browse between images using gestures which are enriched with feedforward animations and interactive feedback.

both sides by placing a hand near the edges of the device. In the detail view, horizontal swipe gestures are employed to switch to the next or previous image. A vertical swipe gesture from top to bottom allows the user to return to the overview.

We implemented various types of visual feedback on the device. When a hand is recognized by the device, a blue glow effect follows the position of the user's hand, similar to a shadow. In the image viewer's overview the regions at both sides of the device are illuminated to visualize the possibility of scrolling (see Figure 6 ). When the hand remains above an image in the overview, the glow effect fades from blue to green to indicate a successful selection. At the time a gesture is performed, the device indicates the successful recognition by shortly lighting up in green (see Figure 7).

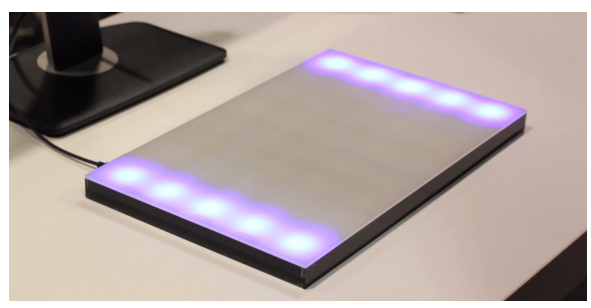

Fig. 6. Interactive regions are visualized with a glow effect. When the hand moves over the corresponding region, an application-specific action is triggered (e.g. scrolling in the image viewer).

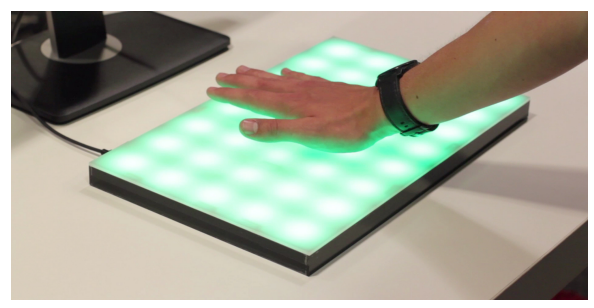

Fig. 7. When a gesture is recognized successfully, the device lights up in green. Moreover, it is possible to indicate unrecognized or unsupported gestures by lighting up in red.

Every participant was instructed to perform a set of tasks, one group obtaining a visual feedback by the device and one without. In order to find out if visual feedback speeds up the interaction $(H 1)$ and makes usage or system 
errors visible faster (H2) we recorded the number of unsuccessfully recognized gestures and the resulting interaction speed by counting the number of actions in a given time span. Additionally, we asked qualitative questions on a Likert scale from 1-10 to investigate if the perception of visual feedback depends on the familarity with a system $\left(H_{4}\right)$ and the interaction becomes easier for novice users (H3).

The participants were asked if they paid attention to the visual feedback provided by the Rainbowfish. One test person did not observe any feedback at all, because she was focused on the application shown on the television. Many other participants had a similar experience: they were not able to interpret the different effects and colors of the board because they focused on the application itself. Some could not associate their actions with a color or animation. Overall, the participants only showed a slight tendency to pay attention to the device's visual feedback (5.65/10 points) and supported them in their initial steps with the device (6.44/10 points). Despite the limited perception of visual feedback, the majority of users did not feel disturbed by the illuminated surface $(3.00 / 10$ points).

In conclusion, the evaluation of a home entertainment application showed that two visual feedback mechanisms - the graphical user interface and the gesture recognizing board itself - were not necessary for the majority of users. Nevertheless, novice users or users who experienced problems during the interaction benefitted from the visual feedback and feedforward animations $\left(H_{4}\right)$. An additional positive aspect can be seen in the influence of the Rainbowfish's multicolor lightning on the intrinsic motivation of a user. It was mentioned by many participants that they liked the device and especially the colors, and were motivated to start interacting with it (H3). The interaction speed could not be increased by providing feedback and feedforward information (H1).

Contactless door-closing mechanism We also conducted an experiment on controlling parts of an intelligent environment without using a graphical user interface. Therefore, Rainbowfish may be incorporated into walls, doors, or home appliances like cooking plates. We built an automatic door that can be controlled using gestures - for example to be used in public restrooms. A user is able to lock, unlock, close and open the door by performing horizontal movements in front of the device. The device delivers interactive feedback on the interaction state and gestures that can be performed. The automatic door control has three possible states, with the related colors: open (green), closed (yellow), and locked (red).

We compared two different types of visual feedback. First, a minimalistic feedback is provided by illuminating the device with the color of the current door state (see Figure 8). Second, we also visualized the gestures that are required to switch to the next state (see Figure 9). For example we visualized a red swipe gesture within the 'closed' state of the door to indicate that the door can be locked. Therefore, the corresponding colors of all states were used to visualize the required gesture. 


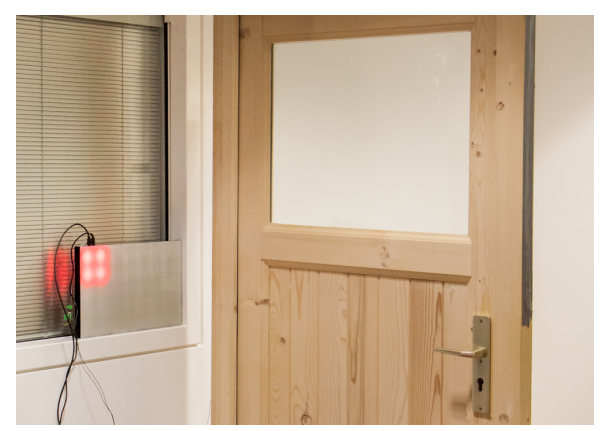

Fig. 8. The minimalistic feedback shows the state of the door - which is currently locked (red).

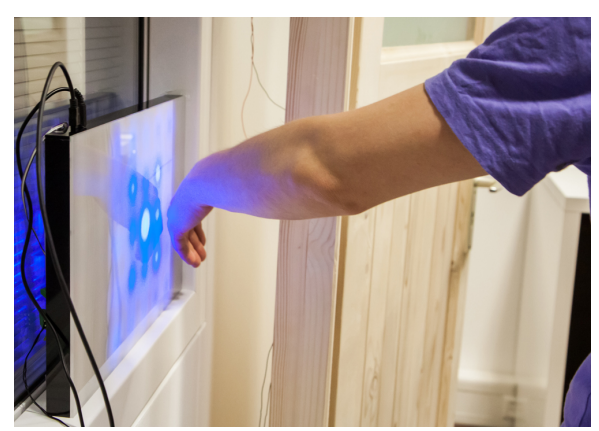

Fig. 9. The extended feedback also indicates when a hand approaches the gesture-recognizing surface.

Rainbowfish's output was essential to recognize the state of the door, as the closed and locked state cannot be differentiated by the user. The participants acknowledged that they directly focused on the visual feedback (8.48/10 points), even if they were not novice users $\left(H_{4}\right)$. At the same time, the users felt slightly more disturbed by the visual feedback than in the first experiment $(3.67 / 10$ points). Nevertheless, most of the participants could interpret the correct meaning of color and animation correctly. However, the interaction speed did not improve (H1). The opinions about the two provided modes varied strongly among the participants. Some of them mentioned that it was not necessary to animate swipe gestures because of their convenience, and a simple state-dependent feedback was sufficient for this use-case. However, the majority of all participants experienced the animated feedback to be very helpful.

\subsection{User Study Summary}

It can be concluded that feedforward animations and feedback can help novice users to control devices by gestures in a smart environment (supporting H2). Visual feedback and feedforward information helps this group of users when experiencing usage problems (support H3). Users who are familiar to a system do not benefit substantially from feedforward animations (supporting $H_{4}$ ). Moreover, the visualizations on Rainbowfish's surface had no influence on the interaction speed (not supporting H1). When providing an additional graphical user interface, the perception of feedback and feedforward animations is very limited. This supports the assumption that a system with visual feedback should be deployed as a stand-alone input modality within a smart environment.

Many users also criticized time delay as well as limited interaction distance. These problems are mainly related to technical issues, which resulted from the transparent electrode material. Mechanical deformations of ITO foil can lead to slight damages of the coating, and thus, a decreased conductivity. This effect resulted in several problems months after building the device. Furthermore, when 
the material is deformed due to mechanical influences (e.g. by a tap on the surface), the capacitance may change rapidly and lead to unexpected behaviour. In the future we will strongly focus on more resilient materials, for example thin conductive layers of silver on PET foil. Also, we aim to achieve interaction distances of $30 \mathrm{~cm}$ increasing the voltage levels from $3.3 \mathrm{~V}$ to $12 \mathrm{~V}$.

\section{Conclusion and Outlook}

In this paper, we presented Rainbowfish, a novel capacitive gesture recognition system capable of delivering interactive visual feedback and feedforward information. The system was implemented with custom-built hardware and a two demonstration applications focusing on different aspects in a smart environment. In a detailed user study, we investigated the usefulness of the proposed method and possible inferences for the usage within a smart environment.

Our user study showed that visual feedback and feedforward information are very helpful for novice users who are not familiar with the corresponding gesture recognition system. When a graphical user interface is employed, experienced users often do not notice visual feedback provided on the gesture recognizing surface. On the other hand, when no GUI is provided, visual feedback also helps experienced users to interact with the gesture-recognizing surface. Having completed the experiments, participants looked forward to use our applications - resulting in a multitude of ideas where technology could be used in the future. Especially public sanitary installations, like toilet flushes, toilet doors or doors in general were mentioned. Besides that, a water tap with a gesture-controlled temperature and water regulation was the most popular idea. Moreover, many applications within a living environment were mentioned, especially in the kitchen and the bathrooms where hygienic requirements are needed. Situations in which the user has sticky hands or carries things can be simplified by gesture-recognizing fridge doors, cookers or drawers. Various other ideas included the control of ambient lightning, gaming, multimedia applications and interactive furniture.

In future work, we will aim at achieving an increased interaction distance, which is currently quite low $(\leq 20 \mathrm{~cm})$. Enhancing the interaction distance will allow for recognizing sophisticated 3D gestures, instead of 2D in-the-air gestures. This possibility raises new research questions on possible visualizations, as a $2 \mathrm{D}$ projection surface is mapped to 3D movements. Moreover, we will work on different types of user feedback, in particular by providing additional sounds when a gesture is recognized.

\section{Acknowledgments}

We would like to thank the students, visitors of the university fair Hobit, employees of Fraunhofer IGD and Technische Universitaet Darmstadt who took part in the user study. 


\section{References}

1. Ballagas, R., Borchers, J., Rohs, M., Sheridan, J.G.: The smart phone: a ubiquitous input device. IEEE Pervasive Computing 5(1), 70-77 (2006)

2. Braun, A., Hamisu, P.: Using the human body field as a medium for natural interaction. In: PETRA '09. pp. 50:1-50:7 (2009)

3. Cohn, G., Morris, D., Patel, S., Tan, D.: Humantenna: using the body as an antenna for real-time whole-body interaction. In: CHI '12. pp. 1901-1910 (2012)

4. Glinsky, A.: Theremin: Ether Music and Espionage. University of Illinois Press (2000)

5. Grosse-Puppendahl, T., Berghoefer, Y., Braun, A., Wimmer, R., Kuijper, A.: Opencapsense: A rapid prototyping toolkit for pervasive interaction using capacitive sensing. In: PerCom '13. pp. 152-159 (2013)

6. Grosse-Puppendahl, T., Braun, A., Kamieth, F., Kuijper, A.: Swiss-cheese extended: an object recognition method for ubiquitous interfaces based on capacitive proximity sensing. In: CHI '13. pp. 1401-1410 (2013)

7. Harrison, C., Sato, M., Poupyrev, I.: Capacitive fingerprinting: exploring user differentiation by sensing electrical properties of the human body. In: UIST '12. pp. $537-544(2012)$

8. Majewski, M., Braun, A., Marinc, A., Kuijper, A.: Providing visual support for selecting reactive elements in intelligent environments. Transactions on Computational Science XVIII 7848, 248-263 (2013)

9. Microsoft: http://www.xbox.com/kinect/, accessed 06/20/2013

10. Poupyrev, I., Yeo, Z., Griffin, J.D., Hudson, S.: Sensing human activities with resonant tuning. In: CHI '10 EA. pp. 4135-4140 (2010)

11. Sato, M., Poupyrev, I., Harrison, C.: Touché: enhancing touch interaction on humans, screens, liquids, and everyday objects. In: CHI '12. pp. 483-492 (2012)

12. Smith, J.R., Gershenfeld, N., Benton, S.A.: Electric Field Imaging. Ph.D. thesis, Massachusetts Institute of Technology (1999)

13. Sodhi, R., Benko, H., Wilson, A.: Lightguide: projected visualizations for hand movement guidance. In: CHI '12. pp. 179-188 (2012)

14. Sousa, M., Techmer, A., Steinhage, A., Lauterbach, C., Lukowicz, P.: Human tracking and identification using a sensitive floor and wearable accelerometers. In: PerCom '13. vol. 18, p. 22 (2013)

15. Valtonen, M., Vuorela, T., Kaila, L., Vanhala, J.: Capacitive indoor positioning and contact sensing for activity recognition in smart homes. JAISE 4, 1-30 (2012)

16. Wimmer, R., Kranz, M., Boring, S., Schmidt, A.: Captable and capshelf - unobtrusive activity recognition using networked capacitive sensors. In: INSS '07. pp. 85-88 (2007) 